\title{
Nitrogen Nuclear Quadrupole Resonance Dips in the Proton Spin Relaxation Dispersion of Nematic and Smectic Thermotropic Liquid Crystals
}

\author{
D. J. Pusiol*, R. Humpfer, and F. Noack \\ Physikalisches Institut, Universität Stuttgart, Stuttgart, FRG \\ Z. Naturforsch. 47a, 1105-1114 (1992); received August 27, 1992
}

\begin{abstract}
The Larmor frequency dependence of the proton spin relaxation time, obtained by means of the fast field-cycling NMR technique, has been used to study the ${ }^{14} \mathrm{~N}$ quadrupole coupling constant $K$ and its asymmetry parameter $\eta$ in the nematic and smectic phases of some liquid crystalline azoxybenzenes (PAA, BAB, HAB, HpAB), cyanobiphenyls (8CB, 9CB, 11 CB) and oxycyanobiphenyls $(9 \mathrm{OCB})$. Due to fast molecular reorientations, the effective quadrupole coupling constants are relatively small, whereas surprisingly the asymmetry parameters are rather large. The temperature dependence of both $K$ and $\eta$ within the mesophases, as well as their discontinuities at the different mesophase transitions, can be interpreted by the anisotropy of molecular rotations. It is found that temperature effects are significantly more pronounced for the (biaxial) smectic-C phase of the heptyloxyazoxybenzene (HpAB) than for the (uniaxial) smectic-A phase of the various investigated cyano- and oxycyanobiphenyls. As a rule, $\eta$ turned out smaller in the smectic than in the nematic state, whereas $K$ has similar values in both phases.
\end{abstract}

\section{Introduction}

The electric field gradient (EFG) at the site of quadrupole nuclei of molecules in the liquid crystalline state is a sensitive measure of the degree of local ordering, and consequently any variation of the molecular anisotropy can be detected by the observation of the related broadening or frequency shift of nuclear quadrupole resonance (NQR) lines [1]. The EFG at a nuclear site seen by such resonance experiments depends not only on the molecular structure and the local bonds, but also on the molecular motions, since fast reorientations average the effective magnitude on the time scale of accessible Larmor frequencies. With the help of a proton-nitrogen nuclear double resonance technique involving standard mechanical cycling of the magnetic field, Seliger et al. [2] could distinguish between uniaxial and biaxial orientational ordering in some smectogen liquid crystals. They found that in uniaxial smectic systems (smectic-A) the asymmetry parameter $\eta$ of the quadrupole coupling constant $K$ was zero within the error limits, but different from zero for biaxial systems (smectic-C, smectic-

* Present address: Universidad Nacional de Córdoba, Facultad de Matematica, Astronomia y Fisica, Laprida 854, 5000 Córdoba, Argentina.

Reprint requests to Prof. Dr. F. Noack, 4. Physikalisches Institut, Universität Stuttgart, Pfaffenwaldring 57, Postfach 8011 40, W-7000 Stuttgart 80, FRG.
H). We demonstrated recently [3] by making use of electronic fast field-cycling techniques, which allow to detect almost arbitrarily low nitrogen quadrupole resonances, that $\eta$ can be finite not only in uniaxial smectics, but even in the nematic phase, and this finding was verified afterwards by Dvinskikh et al. [4]. The present paper gives additional results, in particular data on purely nematic mesogens (some azoxybenzenes), and a more systematic analysis of all ${ }^{14} \mathrm{~N}$ quadrupole transition spectra studied so far (azoxybenzenes, cyanobiphenyls, oxycyanobiphenyls, see Table 1). The azoxybenzenes have two ${ }^{14} \mathrm{~N}$ atoms in the group linking the two benzene rings, the biphenyls have only a single ${ }^{14} \mathrm{~N}$ atom in the cyano side group of one phenyl ring.

\section{Experimental}

The Larmor frequency dependence of the longitudinal proton relaxation time $T_{1}$ (dispersion) was measured for the liquid crystals listed in Table 1 over a broad range $(v=100 \mathrm{~Hz}$ to $100 \mathrm{MHz})$ by means of field-cycling and standard NMR pulsed techniques $[5,6]$. We considered four homologues (PAA, BAB, $\mathrm{HAB}$, and $\mathrm{HpAB}$ ) of the azoxybenzene (AB) series purchased from Eastman-Kodak, three homologues $(8 \mathrm{CB}, 9 \mathrm{CB}$, and $11 \mathrm{CB})$ of the cyanobiphenyl (CB) 


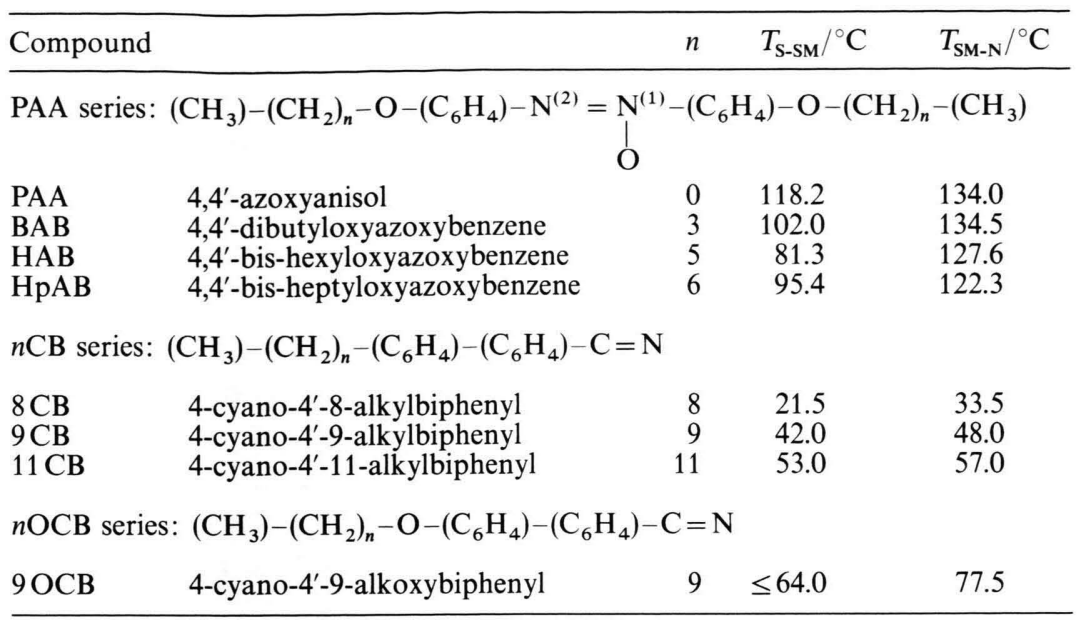

Table 1. Liquid crystal materials of the PAA, $n \mathrm{CB}$ and $n \mathrm{OCB}$ series studied in this work, with phase transition temperatures $T_{\text {S-SM }}$ (solid-smectic) and $T_{\text {SM-N }}$ (smectic-nematic). Note the different ${ }^{14} \mathrm{~N}$ positions in the central group (PAA series) or side group ( $n \mathrm{CB}$ series, $n \mathrm{OCB}$ series), respectively. series and also one member of the oxycyanobiphenyl series (9OCB) obtained from BDH. All these compounds have a nematic mesophase $(\mathrm{N})$; the $\mathrm{nCBs}$ and 9OCB show an additional smectic-A (Sm-A) phase, whereas $\mathrm{HpAB}$ has an additional smectic-C (Sm-C) phase. The materials were sealed in glass tubes by the usual freeze-thaw technique without further purification. In order to orient the director field $\boldsymbol{n}$ in a well-defined geometry relative to the applied Zeeman field $\boldsymbol{B}$, all relaxation measurements were preceded by heating the samples to the isotropic phase with the high magnetic field of the spectrometer on, and then cooled to a selected temperature in the nematic or smectic state, respectively. The quality of the director orientation was examined optically. It was observed that the nitrogen effects were very sensitive to the sample preparation. Details of the experimental procedures and error limits have been described previously $[5,6]$.

\section{Results and Discussion}

\subsection{Experimental Relaxation Dispersion Profiles}

Figures 1-3 illustrate typical $T_{1}(v)$ profiles obtained for some of the studied liquid crystals at selected temperatures. For $9 \mathrm{CB}$ in the $\mathrm{N}$ and Sm-A phase and for $9 \mathrm{OCB}$ in the Sm-A phase (Fig. 1), for PAA in the isotropic (I) and nematic (N) phase (Fig. 2), and finally for $\mathrm{HpAB}$ in the $\mathrm{N}$ and $\mathrm{Sm}-\mathrm{C}$ phase (Figure 3). All the diagrams show the basic features well-known from previous $T_{1}$ field-cycling measurements on these or similar mesogens [3,5-7], namely: (i) a plateau at very low Larmor frequencies, (ii) a strong increase up to about $1 \mathrm{MHz}$ with (iii) the tendency to develop another $T_{1}$ plateau near the crossing point between the data of the isotropic and nematic liquid, and then (iv) the beginning of a high frequency dispersion with increasing slope.

This behaviour is superimposed by several "dips" or "resonances", which are present in all mesophases, but not in the isotropic state. As a rule, the position of the resonances shifts to lower frequencies with increasing temperature, and also involves discontinuities at the phase transitions. The dips are narrower in the nematic than in the smectic samples, and their depth is particularly small in the nematogens without a nematic-tosmectic phase transition.

In order to analyse the $T_{1}(v)$ dips quantitatively, one has to separate the relaxation dipersion due to proton-proton coupling $\left(T_{1 \mathrm{HH}}\right)$ from the effects due to nitrogen spins $\left(T_{1 \mathrm{NH}}\right)$. In the following, we briefly describe the relaxation models considered in the curve fitting to our experimental data and then discuss the significance of the obtained model parameters, with emphasis on the dip frequencies and their relation to ${ }^{14} \mathrm{~N}$ spins.

\subsection{Relaxation Dispersion by Dipolar Proton-Proton Coupling}

For a sake of clarity, the main potential proton relaxation mechanisms in liquid crystals are sketched in Fig. 4 for the PAA molecule (p,p'-azoxyanisole), namely: 

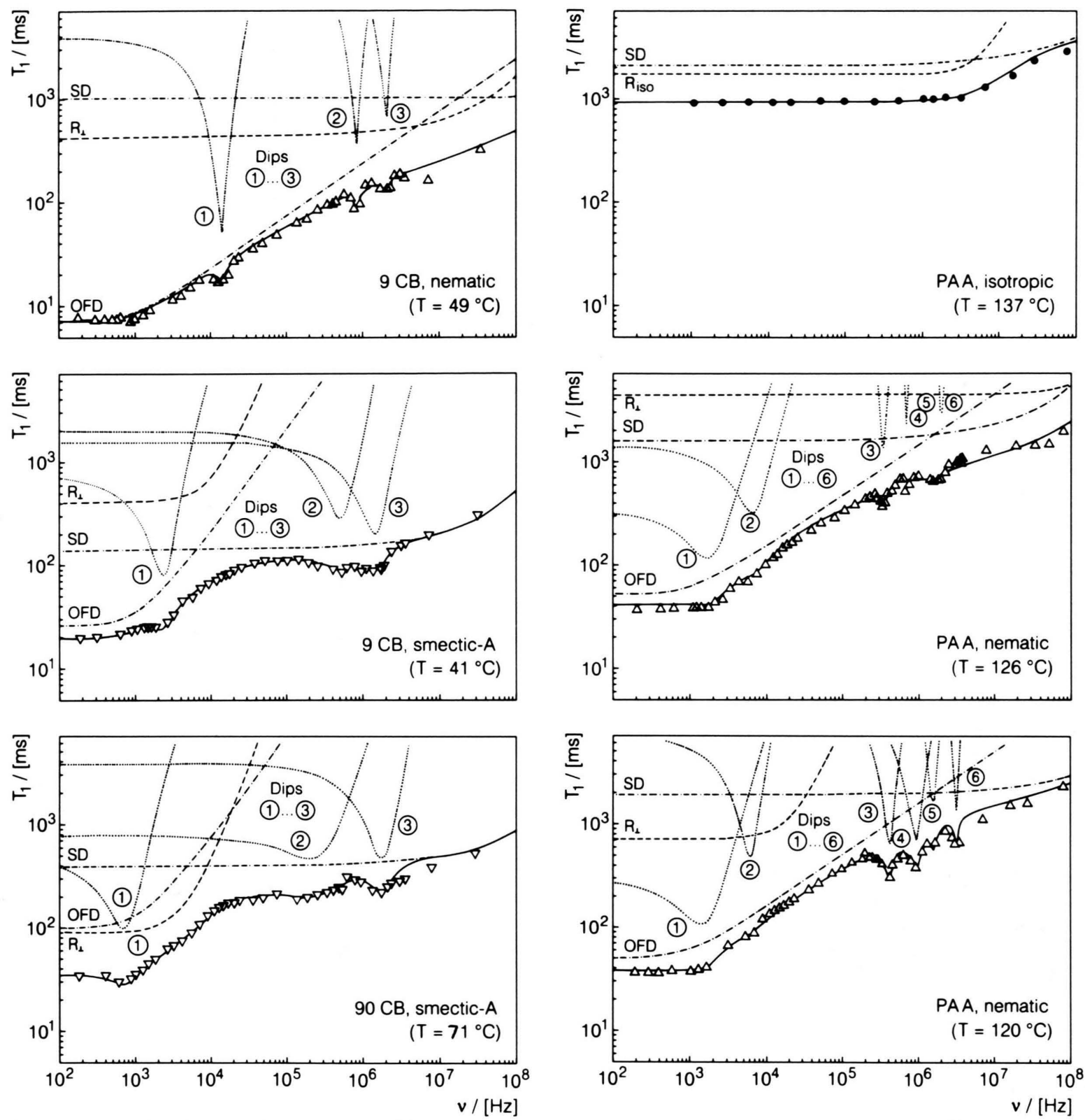

Fig. 1. Proton $T_{1}$ relaxation dispersion with ${ }^{14} \mathrm{~N}$ quadrupole resonances for nematic and smectic-A $9 \mathrm{CB}$, and for smectic-A 9 OCB. The full lines are model fits of Eqs. (1)-(8), the broken lines illustrate the individual contributions to the total relaxation time.

(i) Internal rotations $\left(\mathrm{R}_{\mathrm{I}}\right)$ of molecular groups, and rotational tumbling of the whole molecule parallel to the long axis $\left(\mathbf{R}_{\|}\right)$, or perpendicular to the long axis $\left(\mathbf{R}_{\perp}\right)$.

(ii) Translational self-diffusion (SD) of whole molecules.

Fig. 2. Proton $T_{1}$ relaxation dispersion with ${ }^{14} \mathrm{~N}$ quadrupole resonances for isotropic and nematic PAA. The full lines are model fits of Eqs. (1) - (8), the broken lines illustrate the individual contributions to the total relaxation time.

(iii) Orientational order fluctuations of the director field (OFD), i.e. collective motions of the long molecular axis.

Far away from phase transitions, where critical phenomena come into play, the proton relaxation dis- 

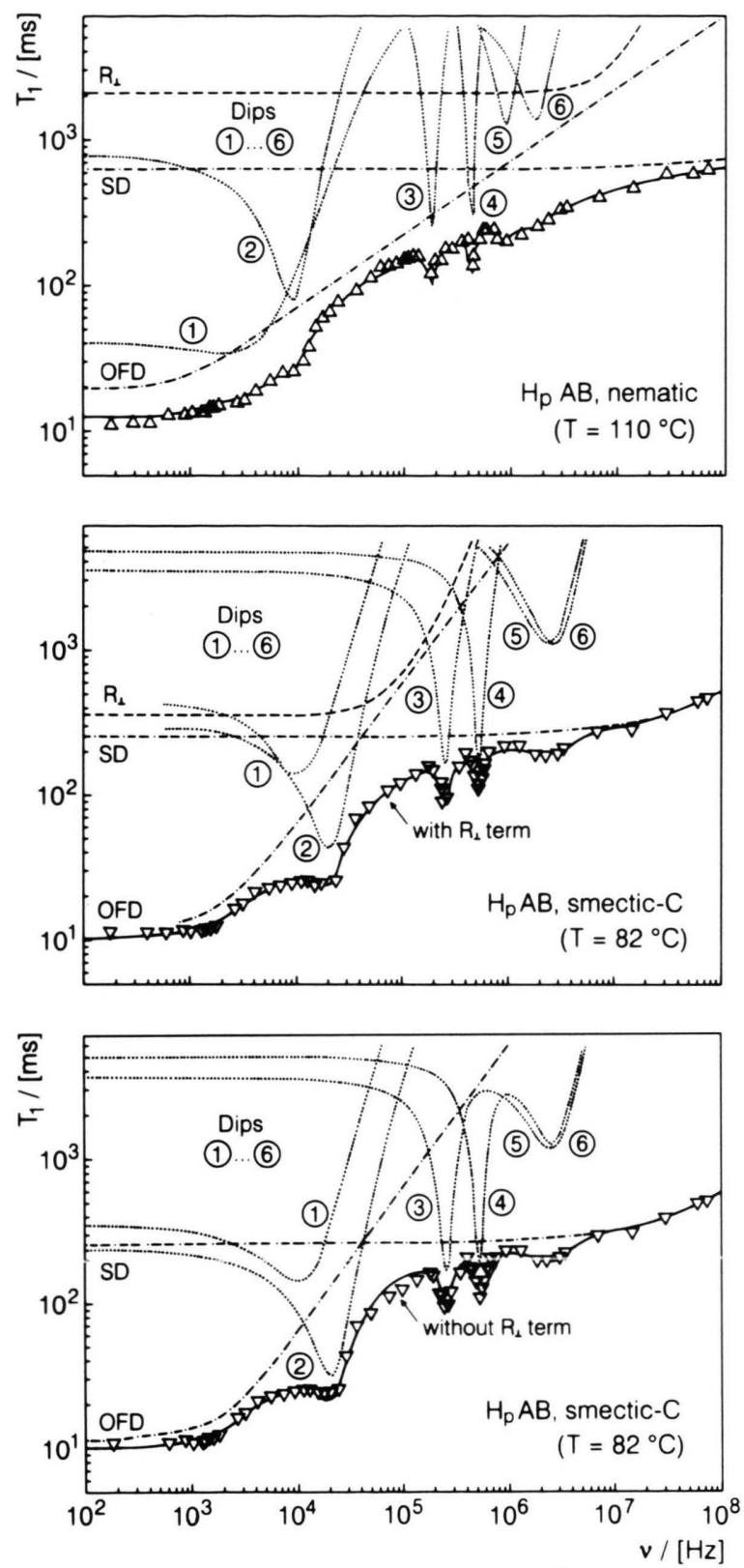

Fig. 3. Proton $T_{1}$ relaxation dispersion with ${ }^{14} \mathrm{~N}$ quadrupole resonances for nematic and smectic-C $\mathrm{HpAB}$. The full lines are model fits of Eqs. (1)-(8), the broken lines illustrate the individual contributions to the total relaxation time. Comparing the two model fits at $82{ }^{\circ} \mathrm{C}$ shows the importance of the rotational $\mathbf{R}_{\perp}$ term.

persion of liquid crystals has been successfully interpreted by adequate superpositions of these mechanisms. Details of the theory are summarized in Table 2. The relative importance of the individual contributions essentially depends on the time scale of the molecular motions relative to the Larmor period, and on the geometry of the spin pair vectors which connect two dipolar coupled proton spins. It was demonstrated in numerous previous studies [5-7] that due to these two criteria, the OFD process [8-10] dominates the relaxation dispersion at low frequencies, typically below $\approx 500 \mathrm{kHz}$, whereas the $\mathrm{SD}$ and $\mathrm{R}_{\perp}$ processes [11-14] determine the high frequency behaviour above $v \approx 5 \mathrm{MHz}$. In accordance with theoretical estimations, both the $R_{I}$ and $R_{\|}$rotations prove too fast to cause a noticeable dispersion step in the accessible Larmor frequency range. The colletice motional OFD modes are usually easily detected by their characteristic dispersion profiles $T_{10 \mathrm{FD}} \sim v^{1 / 2}$ or $T_{10 \mathrm{FD}} \sim v^{1}$, expected for the nematic or smectic mesophase [8-10], respectively. All formulae used to compute the relaxation rates by proton-proton interactions to obtain satisfactory model fits to the experimental data points are given in Table 2, Eqs. (1)-(4).

\subsection{Relaxation Dips by Nitrogen-Proton Cross-Coupling}

The anomalous decrease in the proton relaxation time at special frequencies or the related magnetic field strengths can be attributed to resonances involving the Zeeman levels of the proton $\left({ }^{1} \mathrm{H}\right)$ spins $I$ and the combined quadrupole and Zeeman levels of the nitrogen $\left({ }^{14} \mathrm{~N}\right)$ spins $S$ on the molecules. Following arguments by Kimmich et al. [15, 16], two dip parameters are characteristic for the proton-nitrogen coupling namely:

(i) The Larmor frequencies at which the resonances occur, which reflect the overlapping energy levels of the $I$ and $S$ spins.

(ii) The shapes of the dips in the proton $T_{1}$ dispersion, which essentially reflect the involved relaxation mechanisms.

The pure quadrupole spectrum of the ${ }^{14} \mathrm{~N}$ nuclei consists of three lines, a low frequency line $v_{0}$, and a high frequency doublet, $v_{+}, v_{-}$. These frequencies are determined by the quadrupole coupling constant $K$ and the asymmetry parameter $\eta$. In a magnetic field one expects to observe three resonances (quadrupole dips) corresponding to the Zeeman-perturbed quadrupole spectrum, $v_{0}^{z}, v_{+}^{z}$, and $v_{-}^{z}$. For well-oriented molecules in single crystals or liquid crystals, $v_{0}^{\mathrm{z}}, v_{+}^{\mathrm{z}}$, and $v_{-}^{z}$ are related to $v_{0}, v_{+}$and $v_{-}$and thus to $K$ and $\eta$ by Eq. (5) and Eq. (6) shown in Table $3[15,16]$. 


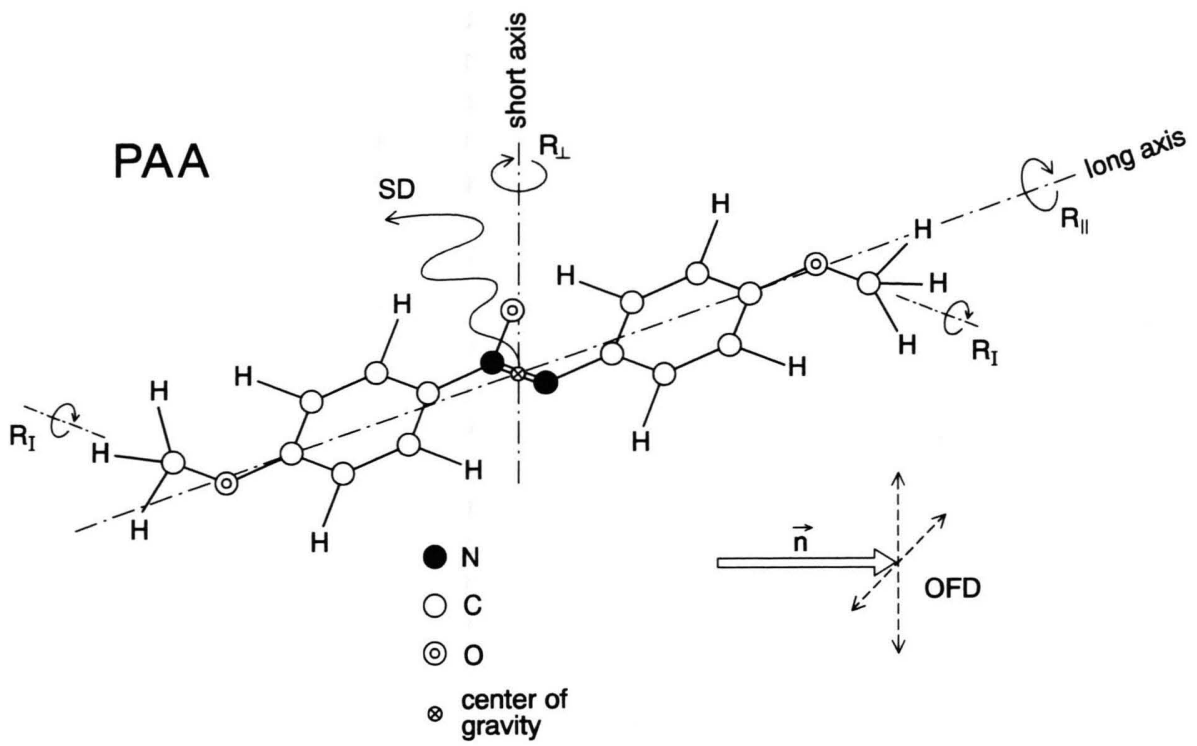

Fig. 4. Schematic representation of the molecular reorientation processes OFD, SD, $R_{\|}, R_{\perp}$ and $R_{I}$ considered by Eq. (8) and the model fits in Figures $1-3$.

Table 2. Model relations used for the curve fittings of $T_{1 \mathrm{HH}}$ in Eq. (8) to the $T_{1}$ dispersion measurements shown in Figs. $1-3$ and to the $T_{1}$ data of all liquid crystals listed in Table 1 . Model fit parameters are the amplitudes $A_{\mathrm{N}}, A_{\mathrm{SM}}, B, C_{1}, C_{2}$, the translational reorientation time $\tau_{\mathrm{SD}}$, the rotational reorientation times $\tau_{\mathbf{R}_{\perp}}, \tau_{\mathbf{R}_{\|}}$and the mode low cut-off frequency $\omega_{\mathbf{c}} / 2 \pi$ $\equiv v_{\mathrm{c}}=1 /\left(2 \pi \tau_{\mathrm{OFD}}\right)$.

\begin{tabular}{lll}
\hline Process & Relaxation rate & Equation Reference \\
\hline
\end{tabular}

Total $T_{1}$ rate $\quad \frac{1}{T_{1 \mathrm{HH}}}=\frac{1}{T_{1 \mathrm{OFD}}}+\frac{1}{T_{1 \mathrm{SD}}}+\frac{1}{T_{1 \mathrm{R}}}$

Nematic OFD $\quad \frac{1}{T_{1 \text { OFD }}}=\frac{A_{\mathrm{N}}}{v^{1 / 2}}\left[1-\frac{1}{\pi}\left[\operatorname{arctg}\left\{\left(2 v / v_{\mathrm{c}}\right)^{1 / 2} /\left(v / v_{\mathrm{c}}-1\right)\right\}-\operatorname{arctg}\left\{\left(2 v / v_{\mathrm{c}}\right)^{1 / 2} /\left(v / v_{\mathrm{c}}+1\right)\right\}\right]\right]+\Theta\left(v / v_{\mathrm{c}}-1\right)$, with $\Theta(v)=0$ for $v<0$ and +1 for $v \geq 0$

Smectic OFD

$$
\frac{1}{T_{1 \mathrm{OFD}}}=\frac{A_{\mathrm{SM}}}{v^{1 / 2}}\left[1-\frac{2}{\pi} \operatorname{arctg}\left(v / v_{\mathrm{c}}\right)\right]
$$

Self-diffusiton (isotropic liquid approximation)

$$
\begin{aligned}
\frac{1}{T_{1 \mathrm{SD}}}=B \tau_{\mathrm{SD}} \sum_{p}^{1,2} p^{2} \frac{\tau_{\mathrm{SD}}}{x^{4}} & {\left[\frac{x}{2}-\frac{1}{x}+e^{-x}\left(\left\{\frac{x}{2}-\frac{1}{x}\right\} \sin x+\left\{\frac{x}{2}+\frac{1}{x}+2\right\} \cos x\right)\right], } \\
\text { with } x & \equiv\left(p 12 \pi v \tau_{\mathrm{SD}}\right)^{1 / 2}
\end{aligned}
$$

Rotations

$$
\frac{1}{T_{1 \mathbf{R}}}=C_{1} \tau_{\mathbf{R}_{\perp}} \sum_{p}^{1,2} p^{2} \frac{1}{\left(1+p 2 \pi \tau_{\mathbf{R}_{\perp}}\right)^{2}}+C_{2} \tau_{\mathbf{R}_{\|}} \quad \text { with } \quad \tau_{\mathbf{R}_{\|}} \ll \tau_{\mathbf{R}_{\perp}}
$$

Both $K$ and $\eta$ are averaged by molecular reorientations of the EFG tensor axes. For example, if one considers ${ }^{14} \mathrm{~N}$ nuclei at positions on the long molecular axis and bonded by an electric charge distribution, with the largest EFG component parallel to that axis and two different perpendicular components $(\eta \neq 0)$, then any reorientation of the molecule around the long axis $\left(\mathrm{R}_{\|}\right)$produces no effects on $K$, but strongly averages $\eta$. On the contrary, rotations about the short axes $\left(\mathrm{R}_{\perp}\right)$ of the rod diminish $K$ but have only small 


\begin{tabular}{llll}
\hline Process & Dip frequencies and relaxation & Eq. & References \\
\hline $\begin{array}{lll}\text { Pure quadrupole } \\
\text { transitions }\end{array}$ & $v_{ \pm}=\frac{3}{4} K\left(1 \pm \frac{1}{3} \eta\right)$ & $(5 \mathrm{a})$ & {$[1,2]$} \\
& $v_{0}=\frac{1}{2} K \eta$ & $(5 \mathrm{~b})$ & \\
$\begin{array}{l}\text { Zeeman perturbed } \\
\text { quadrupole } \\
\text { transitions }\end{array}$ & $v_{+}^{\mathrm{z}}=v_{+}+\frac{1}{3} v_{\mathrm{N}+}^{2}\left(\left(1 / v_{0}\right)+\left(2 / v_{+}\right)+\left(1 / v_{-}\right)\right)$ & $(6 \mathrm{a})$ & {$[15,16]$} \\
& $v_{-}^{\mathrm{z}}=v_{-}+\frac{1}{3} v_{\mathrm{N}-}^{2}\left(-\left(1 / v_{0}\right)+\left(1 / v_{+}\right)+\left(2 / v_{-}\right)\right)$ & $(6 \mathrm{~b})$ & \\
& $v_{0}^{\mathrm{z}}=v_{0}+\frac{1}{3} v_{\mathrm{N} 0}^{2}\left(\left(1 / v_{0}\right)+\left(1 / v_{+}\right)-\left(1 / v_{-}\right)\right)$ & $(6 \mathrm{c})$ & \\
$\begin{array}{l}\text { Lorentzian } \\
\text { NH relaxation } \\
\text { resonance }\end{array}$ & $\frac{1}{T_{1 \mathrm{NH}}}=\frac{1}{T_{1 \mathrm{QD}}}=\sum_{i}^{+,-, 0} E_{i} \frac{\tau_{i}}{1+\left(2 \pi\left(v_{\mathrm{N} i}^{\mathrm{z}}-v_{\mathrm{H}}\right) \tau_{i}\right)^{2}}$ & $(7)$ & {$[5,6,16]$} \\
\hline
\end{tabular}

Table 3. Zeeman-perturbed ${ }^{14} \mathrm{~N}-{ }^{1} \mathrm{H}$ quadrupole level crossing frequencies $v_{0}^{z}, v_{+}^{z}, v_{-}^{z}$, and the related $T_{1 \mathrm{NH}}(v)$ model (Lorentzian) $[1,15,16]$ used for the curve fittings of Eq. (8) to our $T_{1}$ dispersion measurements. Model fit parameters are the dip amplitudes $E_{i}$, the dip frequencies $v_{i}^{z} \equiv v_{\mathrm{N} i}^{z}$, and the widths $\Delta v_{i}=1 /\left(2 \pi \tau_{i}\right)$. $\mathrm{N}$ denotes nitrogen, $\mathrm{H}$ means proton, and $\gamma_{\mathrm{N}}, \gamma_{\mathrm{H}}$ are the related gyromagnetic ratios. effects on $\eta$. Such changes should become most pronounced if the correlation time of the motion coincides with one of the inverse Zeeman-perturbed quadrupole frequencies, in which case the dip positions are very sensitive to temperature shifts.

The shape of the dips depends on the one hand on the shape of the ${ }^{1} \mathrm{H}$ and ${ }^{14} \mathrm{~N}$ NMR spectra, and on the other hand on the involved relaxation processes. In the solid state approximation (short $T_{2}$, spin temperature concept), Kimmich et al. [14, 15] have discussed these relaxation mechanisms in detail, namely the mixture of these energy transfers from the protons to the lattice, from the nitrogen to the lattice and between the protons and nitrogens. In the general casc, this superposition entails a non-exponential recovery of the ${ }^{1} \mathrm{H}$ magnetization to its equilibrium value when the ${ }^{1} \mathrm{H}$ levels nearly overlap with the quadrupole ${ }^{14} \mathrm{~N}$ levels. However, there exist three special, limiting cases with exponential relaxation, where two of the transfer rates are ineffective or negligible compared with the third one. This kind of behaviour was approximately observed in all our relaxation studies over the dip regimes (though minor non-exponential effects were detectable and often used to find the dip positions!). Under such conditions, the $T_{1}(v)$ resonances exhibit both Lorentzian and Gaussian contributions. In view of the relatively small influence of the nitrogen spins on the overall proton relaxation in liquid crystals and the related large error limits over the dip range, our present discussion is restricted to the Lorentzian dip shape (Table 3, Eq. (7)) and does not separate statical from dynamical broadening $[15,16]$.

\subsection{Curve Fittings and Interpretation}

The model fits of the selected theoretical expression

$$
\begin{aligned}
\frac{1}{T_{1}(v)} & =\frac{1}{T_{1 \mathrm{HH}}}+\frac{1}{T_{1 \mathrm{NH}}} \\
& =\frac{1}{T_{1 \mathrm{OFD}}}+\frac{1}{T_{1 \mathrm{SD}}}+\frac{1}{T_{1 \mathrm{R}}}+\frac{1}{T_{1 \mathrm{QD}}}
\end{aligned}
$$

with the individual contributions defined by (1)-(7) (Tables 2 and 3) were obtained by standard leastsquares optimization procedures.

For all studied systems, the $T_{1 \mathrm{HH}}$ term shows properties to be expected from previous measurements on similar liquid crystals. At medium frequencies, $T_{1 \text { OFD }}$ varies proportional to $v^{1 / 2}$ in nematic samples (e.g. PAA, HpAB, 9CB), whereas the smectic samples (e.g. $\mathrm{HpAB}, 9 \mathrm{CB}, 9 \mathrm{OCB})$ reveal a stronger $T_{1 \mathrm{OFD}}$ dispersion, approximately $T_{1 \mathrm{OFD}} \sim v^{1}$, in accordance with the Pincus-Blinc theory [8-10]. However, due to the overlap with the $T_{1 \mathrm{SD}}$ and $T_{1 \mathrm{R}}$ terms which strongly shorten the range where $T_{1 \mathrm{OFD}}$ is effective in the smectic phase, the exponent $n=1$ is less accurate $( \pm 0.35)$ than the exponent $n=0.5( \pm 0.15)$ for the nematic phase. The low-frequency $T_{1}(v)$ plateau can be assigned to the long-wavelength cut-off the overdamped hydrodynamic collective modes. At high Larmor frequencies, the data could be fitted by using a combination of the Pfeifer-Abragam $T_{1 \text { sD }}$ model in the isotropic small-step apporoximation [11] with the $T_{1 \mathrm{R}}$ model of Woessner [14] for anisotropic rotations of the whole molecule around the short axis $\left(R_{\perp}\right)$. One should note 
Table 4. Relaxation model fit parameters for the $T_{1 \mathrm{HH}}$ term in Eq. (8) (see Table 2). The separation of the rotation and self-diffusion processes is not possible unambiguously.

\begin{tabular}{|c|c|c|c|c|c|c|c|c|}
\hline Compound & $T /\left[{ }^{\circ} \mathrm{C}\right]$ & $A_{\mathrm{N}} /\left[\mathrm{s}^{-3 / 2}\right]$ & $A_{\mathrm{SM}} /\left[\mathrm{s}^{-2}\right]$ & $v_{\mathrm{c}} /[\mathrm{kHz}]$ & $B /\left[\mathrm{s}^{-2}\right]$ & $\tau_{\mathrm{SD}} /[\mathrm{ns}]$ & $C_{1} \tau_{\perp} /\left[\mathrm{s}^{-1}\right]$ & $\tau_{\perp} /[\mathrm{ps}]$ \\
\hline PAA & $\begin{array}{l}126(\mathrm{~N}) \\
120(\mathrm{~N})\end{array}$ & $\begin{array}{l}605 \\
590\end{array}$ & - & $\begin{array}{l}0.9 \\
0.8\end{array}$ & $\begin{array}{l}1.3 \cdot 10^{9} \\
1.4 \cdot 10^{9}\end{array}$ & $\begin{array}{l}0.20 \\
0.15\end{array}$ & $\begin{array}{l}0.22 \\
1.4\end{array}$ & $\begin{array}{l}<\tau_{\mathrm{SD}} \\
>\tau_{\mathrm{SD}}\end{array}$ \\
\hline НрАВ & $\begin{aligned} 110(\mathrm{~N}) \\
82(\mathrm{Sm}-\mathrm{C})\end{aligned}$ & $\begin{array}{r}1600 \\
-\end{array}$ & $\overline{1.5} \cdot 10^{5}$ & $\begin{array}{r}4 \\
10\end{array}$ & $\begin{array}{l}3.3 \cdot 10^{9} \\
4.2 \cdot 10^{9}\end{array}$ & $\begin{array}{l}0.20 \\
0.40\end{array}$ & $\begin{array}{l}0.47 \\
2.6\end{array}$ & $\begin{array}{l}<\tau_{\mathrm{SD}} \\
\cong 900\end{array}$ \\
\hline $9 \mathrm{CB}$ & $\begin{array}{l}49(\mathrm{~N}) \\
41(\mathrm{Sm}-\mathrm{A})\end{array}$ & $\begin{array}{r}4500 \\
-\end{array}$ & $3.2 \cdot 10^{5}$ & $\begin{array}{r}7 \\
10\end{array}$ & $\begin{array}{l}1.1 \cdot 10^{9} \\
6.2 \cdot 10^{9}\end{array}$ & $\begin{array}{l}0.4 \\
0.5\end{array}$ & $\begin{array}{l}2.3 \\
2.5\end{array}$ & $\begin{array}{l}>\tau_{\mathrm{SD}} \\
>\tau_{\mathrm{SD}}\end{array}$ \\
\hline 9 OCB & 71 (Sm-A) & - & $1.2 \cdot 10^{5}$ & 7 & $2.6 \cdot 10^{9}$ & 0.4 & 10 & $>\tau_{\mathrm{SD}}$ \\
\hline
\end{tabular}

that the dramatic broadening of the high-frequency dispersion step in the smectic compared with the nematic mesophase reflects the much slower molecular motions (longer reorientation times) for the smectic, i.e. the lower temperature ordering. Yet inspite of the increased importance of the $T_{1 \mathrm{SD}}$ and $T_{1 \mathrm{R}}$ terms, the available Larmor frequency range at the moment is still insufficient to separate these two contributions reliably alone by the observed $T_{1}$ dispersion. The best fit parameters are listed in Table 4.

Over each dip regime, $T_{1}(v)$ was fitted by a Lorentzian function for $1 / T_{1 \mathrm{QD}}$ (Eq. (7)). The observed three quadrupole dips (QD) in the Sm- and $\mathrm{N}$-phases of the cyanobiphenyls and oxycyanobiphenyls indicate a three line, Zeeman-perturbed, quadrupole spectrum $\left(v_{0}^{z}, v_{+}^{z}, v_{-}^{z}\right)$ of the ${ }^{14} \mathrm{~N}$ spins in these molecules. Since the high-frequency doublets are positioned in the range between 1 and $2 \mathrm{MHz}$, with $\eta=0$ the Zeeman splitting of the ${ }^{14} \mathrm{~N}$ quadrupole levels calculated by (5) results to less than $100 \mathrm{kHz}$. However, the observed splittings between $v_{+}^{z}$ and $v_{-}^{z}$ are much larger than $100 \mathrm{kHz}$ in all the analysed compounds (for instance, $850 \mathrm{kHz}$ for Sm-A 9 CB and $960 \mathrm{kHz}$ for N 9 CB). This fact, together with the occurrence of the low-frequency QD at $v_{0}^{z}$, strongly suggests that $\eta$ must be non-zero in both the $\mathrm{Sm}$ and $\mathrm{N}$ mesophases. The same argument holds in all the compounds of the CB, OCB and PAA series. Tables $5 \mathrm{a}$ and $5 \mathrm{~b}$ show the model fit constants.

For the nematic azoxybenzenes, five quadrupole dips were observed. There exists a low-frequency doublet, typically near $5 \mathrm{kHz}$, and a high-frequency triplet, typically near $2 \mathrm{MHz}$. In the following, the individual lines will be denoted by $v_{i}$, with $i=1, \ldots, 5$, starting from the lowest line. Obviously, the finding $i>3$ is related to the nonequivalent bonds and the different EFG tensors of the two ${ }^{14} \mathrm{~N}$ nuclei of the molecule
[17]. To assign the unlike sites to the 5 measured dip frequencies, let $\mathrm{N}^{(1)}$ be the ${ }^{14} \mathrm{~N}$ bonded to the oxygen atom and $\mathrm{N}^{(2)}$ be the other one [8] (see Fig. 4 for the schematic molecular structure of PAA). It is reasonable to expect $K^{(2)}$ to be greater than $K^{(1)}$ and $\eta^{(2)}$ to be smaller than $\eta^{(1)}$ for the two ${ }^{14} \mathrm{~N}$ sites, if one compares our results with the interpretation of the pure ${ }^{14} \mathrm{~N}$ NQR spectrum of solid PAA and HAB reported in the literature [19]. We further assume that the dip with frequency $v_{5}$ is actually produced by a pair of unresolved high-frequency dips with very small asymmetry parameter $\eta$, whereas the finite splitting between $v_{3}$ and $v_{4}$ may be a consequence of a somewhat larger, non-zero value of $\eta$. Considering the same aspect, we relate the unresolved $v_{5}$ doublet to the lowest dip at $v_{1}$. Consequently, dips with frequencies $v_{2}, v_{3}$, $v_{4}$ should be assigned to the $\mathrm{N}^{(2)}$ nuclei and the dips $v_{1}, v_{5}$, to $\mathrm{N}^{(1)}$. However, due to the error limits, it is also possible to relate $v_{5}$ with the dip frequency $v_{2}$.

The values of $K$ and $\eta$ obtained from the fitted relaxation dispersion at several temperatures are listed in Table $5 \mathrm{a}$. For the biphenyls in the Sm-A phase, $K$ is only slightly temperature dependent (1.1 to $1.5 \mathrm{MHz}$ ), while $\eta$, although of very small magnitude $(0.4 \%$ at most $)$, reveals stronger changes. In these compounds the long molecular axis has almost the same direction as the largest EFG component, a geometry for which fast uniaxial rotations about the long axis should yield temperature independent $K$ values and $\eta \simeq 0$ [1]. The observed non-zero $\eta$ 's demonstrate that molecular rotations around the long axis are not completely isotropic, i.e. biased perpendicular to the rotation, corresponding to a weak local transverse ordering. Through the $\mathrm{Sm}-\mathrm{A}$ to $\mathrm{N}$ transition, $\eta$ is seen to increase suddenly by more than one order of magnitude, but $K$ remains almost unchanged. Two features can be seen from this behaviour: First, similar 
Table 5a. Relaxation model fit parameters for the $T_{1 \mathrm{NH}}$ term in Eq. (8) (see Table 3): Quadrupole coupling constants $K^{(j)}$, and asymmetry parameters $\eta^{(j)}, j=1,2$.

\begin{tabular}{|c|c|c|c|c|c|c|}
\hline \multirow{2}{*}{$\begin{array}{l}\text { Com- } \\
\text { pound }\end{array}$} & \multirow[t]{2}{*}{$T /\left[{ }^{\circ} \mathrm{C}\right]$} & \multirow[t]{2}{*}{ Phase } & \multicolumn{2}{|c|}{$K /[\mathrm{kHz}]$} & \multicolumn{2}{|c|}{$\eta /[\%]$} \\
\hline & & & $K^{(1)}$ & $K^{(2)}$ & $\eta^{(1)}$ & $\eta^{(2)}$ \\
\hline
\end{tabular}

\section{PAA series}

$\begin{array}{lrlrrrr}\text { PAA } & 125.5 & \mathrm{~N} & 2100 & 600 & 0.15 & 2.00 \\ & 120.5 & \mathrm{~N} & 4087 & 853 & 0.08 & 1.40 \\ \text { BAB } & 131.0 & \mathrm{~N} & 1733 & 647 & 0.15 & 2.16 \\ & 124.0 & \mathrm{~N} & 2100 & 760 & 0.18 & 1.90 \\ & 117.0 & \mathrm{~N} & 1967 & 860 & 0.21 & 1.67 \\ \text { HAB } & 114.5 & \mathrm{~N} & 2067 & 567 & 0.19 & 1.69 \\ \text { HpAB } & 115.0 & \mathrm{~N} & 2267 & 303 & 0.16 & 6.30 \\ & 110.0 & \mathrm{~N} & 1533 & 397 & 0.34 & 4.79 \\ & 93.0 & \mathrm{Sm}-\mathrm{C} & 2300 & 77 & 0.11 & 35.0 \\ & 89.0 & \text { Sm-C } & 2133 & 120 & 0.21 & 14.0 \\ & 82.0 & \text { Sm-C } & 3267 & 507 & 1.41 & 5.13 \\ & 75.0 & \text { Sm-C } & 3600 & 1360 & 6.57 & 2.20\end{array}$

$n C B$ and $n O C B$ series

\begin{tabular}{lrlrl}
$8 \mathrm{CB}$ & 32.0 & $\mathrm{~N}$ & 933 & 2.68 \\
& 24.0 & $\mathrm{~N}$ & 1600 & 1.75 \\
& 23.0 & $\mathrm{Sm}-\mathrm{A}$ & 1453 & 0.07 \\
& 9.5 & $\mathrm{Sm}-\mathrm{A}$ & 1600 & 0.21 \\
\multirow{2}{*}{$\mathrm{CB}$} & 49.0 & $\mathrm{~N}$ & 1773 & 1.70 \\
& 45.0 & $\mathrm{Sm}-\mathrm{A}$ & 1833 & 0.17 \\
& 41.0 & $\mathrm{Sm}-\mathrm{A}$ & 1300 & 0.39 \\
$11 \mathrm{CB}$ & 55.0 & $\mathrm{Sm}-\mathrm{A}$ & 1080 & 0.20 \\
9 OCB & 71.0 & $\mathrm{Sm}-\mathrm{A}$ & 1133 & 0.14 \\
\hline
\end{tabular}

values of $K$ in both phases indicate that the fluctuation rates of the long molecular axis are practically the same; secondly, the drop in $\eta$ suggests the surprising result that the molecules rotate "more freely" in the Sm-A than in the $\mathrm{N}$ phase.

For the azoxybenzenes, the temperature behaviour of $K^{(1)}, K^{(2)}, \eta^{(1)}$ and $\eta^{(2)}$ shows parallels but also notable distinctions to all the biphenyls. In the $\mathrm{N}$ phase the quadrupole coupling constants are almost temperature independent, while the $\eta$ 's vary a little more strongly. Hence the same analysis is suitable in both kinds of mesogens. In other words, the EFG at the nitrogen sites $\mathrm{N}^{(1)}$ and $\mathrm{N}^{(2)}$ of nematic $A B$ 's is dynamically averaged in a similar way than the EFG at the $\mathrm{N}$ site of the CB's. In accordance with this model, the phase transition from $\mathrm{N}$ to $\mathrm{Sm}-\mathrm{C}$ for $\mathrm{HpAB}$ happens without a significant change of $K^{(2)}$, i.e. in the same manner as shown by $K$ for the CB's at the $\mathrm{N}$ to Sm-A transition. In contrast to this, $K^{(1)}$ jumps by one order of magnitude at the transition, and the discontinuity is followed by an anomalous, spectacular tem-
Table $5 \mathrm{~b}$. Relaxation model fit parameters for the $T_{1 \mathrm{NH}}$ term in Eq. (8) (see Table 3): Dip intensities $E_{i}$, dip frequencies $v_{i}$, and dip width parameters $\tau_{i}, i=1, \ldots, 6$ (PAA series) and $i=1, \ldots, 3$ ( $n \mathrm{CB}$ and $n \mathrm{OCB}$ series).

Com- $T /\left[{ }^{\circ} \mathrm{C}\right]$ Phase $i \quad E_{i} /\left[10^{4} \mathrm{~s}^{-1}\right] v_{i} /[\mathrm{kHz}] \tau_{i} /[\mu \mathrm{s}]$ pound

\begin{tabular}{|c|c|c|c|c|c|c|}
\hline PAA & 120 & $\mathrm{~N}$ & $\begin{array}{l}1 \\
2 \\
3 \\
4 \\
5 \\
6\end{array}$ & $\begin{array}{r}9 \\
8 \\
70 \\
175 \\
100 \\
125\end{array}$ & $\begin{array}{r}1.6 \\
6.0 \\
400 \\
880 \\
2900 \\
3200\end{array}$ & $\begin{array}{r}110 \\
40 \\
2.5 \\
0.8 \\
0.5 \\
0.4\end{array}$ \\
\hline $\mathrm{BAB}$ & 117 & $\mathrm{~N}$ & $\begin{array}{l}1 \\
2 \\
3 \\
4 \\
5 \\
6\end{array}$ & $\begin{array}{r}12 \\
70 \\
65 \\
700 \\
80 \\
55\end{array}$ & $\begin{array}{r}2.3 \\
6.0 \\
460 \\
830 \\
1400 \\
1550\end{array}$ & $\begin{array}{r}100 \\
22 \\
15 \\
250 \\
2 \\
1.5\end{array}$ \\
\hline HAB & 115 & $\mathrm{~N}$ & $\begin{array}{l}1 \\
2 \\
3 \\
4 \\
5 \\
6\end{array}$ & $\begin{array}{r}35 \\
50 \\
35 \\
150 \\
225 \\
250\end{array}$ & $\begin{array}{r}2.0 \\
4.8 \\
275 \\
575 \\
1350 \\
1750\end{array}$ & $\begin{array}{c}40 \\
30 \\
15 \\
2 \\
0.4 \\
0.3\end{array}$ \\
\hline \multirow[t]{2}{*}{$\mathrm{HpAB}$} & 110 & $\mathrm{~N}$ & $\begin{array}{l}1 \\
2 \\
3 \\
4 \\
5 \\
6\end{array}$ & $\begin{array}{r}30 \\
45 \\
60 \\
70 \\
100 \\
110\end{array}$ & $\begin{array}{c}0.4 \\
9 \\
180 \\
420 \\
1100 \\
1300\end{array}$ & $\begin{array}{l}14 \\
22 \\
50 \\
40 \\
16 \\
16\end{array}$ \\
\hline & 82 & $\mathrm{Sm}-\mathrm{C}$ & $\begin{array}{l}1 \\
2 \\
3 \\
4 \\
5 \\
6\end{array}$ & $\begin{array}{l}100 \\
175 \\
150 \\
225 \\
900 \\
900\end{array}$ & $\begin{array}{r}13 \\
23 \\
250 \\
510 \\
2300 \\
2600\end{array}$ & $\begin{array}{r}9 \\
16 \\
4 \\
2 \\
0.1 \\
0.1\end{array}$ \\
\hline \multirow[t]{2}{*}{$8 \mathrm{CB}$} & 32 & $\mathrm{~N}$ & $\begin{array}{l}1 \\
2 \\
3\end{array}$ & $\begin{array}{r}30 \\
300 \\
1500\end{array}$ & $\begin{array}{c}12.5 \\
400 \\
1000\end{array}$ & $\begin{array}{l}52 \\
2 \\
0.8\end{array}$ \\
\hline & 23 & Sm-A & $\begin{array}{l}1 \\
2 \\
3\end{array}$ & $\begin{array}{r}150 \\
1800 \\
1200\end{array}$ & $\begin{array}{c}0.5 \\
680 \\
1500\end{array}$ & $\begin{array}{c}180 \\
0.5 \\
2\end{array}$ \\
\hline \multirow[t]{2}{*}{$9 \mathrm{CB}$} & 49 & $\mathrm{~N}$ & $\begin{array}{l}1 \\
2 \\
3\end{array}$ & $\begin{array}{r}50 \\
400 \\
550\end{array}$ & $\begin{array}{r}15 \\
850 \\
1810\end{array}$ & $\begin{array}{r}46 \\
1 \\
2\end{array}$ \\
\hline & 41 & Sm-A & $\begin{array}{l}1 \\
2 \\
3\end{array}$ & $\begin{array}{r}15 \\
1200 \\
2200\end{array}$ & $\begin{array}{r}2.3 \\
450 \\
1300\end{array}$ & $\begin{array}{c}86 \\
0.5 \\
0.3\end{array}$ \\
\hline 9 ОСВ & 71 & Sm-A & $\begin{array}{l}1 \\
2 \\
3\end{array}$ & $\begin{array}{r}5 \\
300 \\
550\end{array}$ & $\begin{array}{c}0.8 \\
200 \\
1500\end{array}$ & $\begin{array}{r}160 \\
0.6 \\
0.3\end{array}$ \\
\hline $11 \mathrm{CB}$ & 55 & Sm-A & $\begin{array}{l}1 \\
2 \\
3\end{array}$ & $\begin{array}{r}30 \\
400 \\
450\end{array}$ & $\begin{array}{c}1.1 \\
420 \\
1400\end{array}$ & $\begin{array}{r}90 \\
1 \\
2\end{array}$ \\
\hline
\end{tabular}



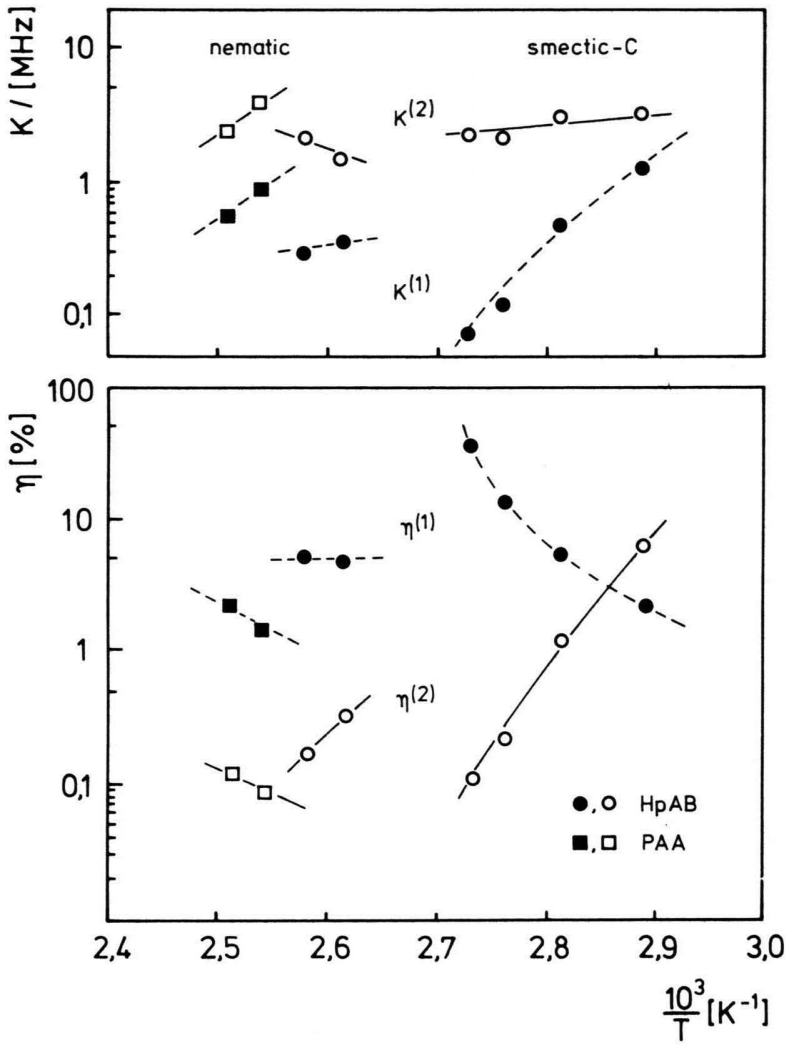

Fig. 5. Temperature dependence of the quadrupole coupling constants $K^{(i)}$ and asymmetry parameters $\eta^{(i)}$ obtained for PAA (nematic) and HpAB (nematic, smectic-C).

perature dependence in the $\mathrm{Sm}-\mathrm{C}$ mesophase. We ascribe this special effect not observed in the other systems to the peculiar Sm-C geometry and the related, heavily hindered molecular rotation about the short axis $\left(\mathbf{R}_{\perp}\right)$. As illustrated by the individual relaxation terms of the $T_{1}(v)$ dispersion profile for $\mathrm{HpAB}$ (Fig. 3), this reorientation also influences $T_{1}$, in addition to the two dominating $T_{1 \mathrm{OFD}}$ and $T_{1 \mathrm{SD}}$ terms. The main correction of the $R_{\perp}$ process to the overall model fit occurs at frequencies near the high-field quadrupole dip-pairs, and yields motional correlation times of $2.4 \mu$ s and $0.9 \mu$ s at 75 and $82^{\circ} \mathrm{C}$, respectively, i.e. a large activation energy of approximately $140 \mathrm{~kJ} / \mathrm{mol}$.

Lotfullin and Semin [20] have extensively studied the importance of such rotations on the temperature dependence of the quadrupole coupling constant. For nuclei with spin $I=1$ as ${ }^{14} \mathrm{~N}$ one expects a temperature averaging law of the form [21] $K(T)$ $\sim \operatorname{arctg}\left[\alpha K_{0} \tau_{\mathrm{c}}(T)\right]$, where $\alpha$ is a constant close to unity, $K_{0}$ the quadrupole coupling constant in the absence of molecular rotations, and $\tau_{\mathrm{c}}(T)$ the correlation time of the motion. Assuming that the correlation time of the $R_{\perp}$ motions follows an Arrhenius law, the measured dip frequency shifts and (5) give an activation energy of $170 \mathrm{~kJ} / \mathrm{mol}$. This value agrees well with the result obtained from $T_{1}(v)$ model fits to the dispersion profiles.

Figure 5 contrasts the strong variations of the Sm-C $\mathrm{HpAB}$ quadrupole coupling constants (or the related dip frequencies) with the typically minor changes for nematics. For example, $K^{(2)}\left(\hat{=} v_{3}, v_{4}\right)$ of $\mathrm{HpAB}$ decreases from $1360 \mathrm{kHz}$ at $75^{\circ} \mathrm{C}$ to $77 \mathrm{kHz}$ at $93{ }^{\circ} \mathrm{C}$, whereas $K^{(1)}\left(\hat{=} v_{5}\right)$, similar to $\mathrm{K}$ in $8 \mathrm{CB}$, is nearly constant $(\approx 2200 \mathrm{kHz})$, so that the dip doublets approach each other at low temperatures. Together with the opposite temperature dependence of $\eta^{(1)}$ and $\eta^{(2)}$, these findings support our assumption to correlate $v_{1}$ with the unresolved line $v_{5}$, and $v_{2}$ with the $v_{3}-v_{4}$ pair, respectively.

\section{Summary and Conclusions}

This study has presented further experimental evidences and a quantitative analysis of the protonnitrogen ${ }^{1} \mathrm{H}-{ }^{14} \mathrm{~N}$ cross-coupling in several liquid crystals with nitrogen spins, which in addition to the familiar proton-proton relaxation mechanisms (molecular rotations, self-diffusion, director order fluctuations) contributes to the overall relaxation dispersion $T_{1}(v)$ by narrow "quadrupole" dips at characteristic, low and medium Larmor frequencies. Surprisingly, the dips occur in both the nematic and smectic states of the considered systems, and disappear only in the isotropic liquid. Their number clearly demonstrates small asymmetries of the electric field gradient for the proton-nitrogen bond of the involved nitrogen group. Therefore, on the NMR time scale, one has to assume a "small degree of biaxiality" of the molecular rotations about the long axis, which was not observed previously by other methods.

The measured values of the asymmetry parameters are comparable for the nematic and smectic-A phases and somewhat larger for smectic-C HpAB, but in any case up to two orders of magnitude smaller than data reported by Seliger et al. for higher ordered, solid like mesogen states [1, 2] of the liquid crystal terephtal-bisp-butylaniline (TBBA). Both the quadrupole coupling constant and its asymmetry are very sensitive to the site of the nitrogen spin and to the molecular ordering, 
so that the smectic-C and smectic-A symmetries are easy to distinguish. However, till now spectral resolution and stability of our field-cycling technique are not yet sufficient to learn more details about the "biaxiality", which in principle are contained in the form of the cross-relaxation resonance. Adequate apparative improvements are in progress. We also consider measurements on liquid crystals with other quadrupole nuclei like chlorine, because such a step makes accessible quite different, less symmetric bonds on the molecules.

\section{Acknowledgements}

The authors thank Dr. K. H. Schweikert for the helpful discussions on experimental problems. D.P. particularly acknowledges the research fellowship in Germany by the Alexander von Humboldt Foundation. This work was partially supported by the Deutsche Forschungsgemeinschaft.
[1] R. Blinc, M. Vilfan, and J. Seliger, Bull. Magn. Reson. 5, 51 (1983).

[2] (a) J. Seliger, R. Osredkar, V. Žagar, and R. Blinc, Phys. Rev. Lett. 38, 411 (1977). - (b) J. Seliger, V. Žagar, and R. Blinc, Phys. Rev. A 17, 1149 (1978).

[3] D. Pusiol and F. Noack, Liq. Cryst. 5, 377 (1989).

[4] S. Dvinskikh and Y. Molchanov, Kimicheskaya Fizika 10, 1204 (1991).

[5] F. Noack, Prog. Nucl. Magn. Reson. Spectrosc. 18, 171 (1986).

[6] D. Pusiol and F. Noack, Proc. 10th Ampere Summer School in Portorož (R. Blinc, M. Vilfan, and J. Slak, eds.), Portorož, Yugoslavia, p. 222 (1988).

[7] F. Noack, M. Notter, and W. Weiss, Liq. Cryst. 3, 907 (1988).

[8] P. Pincus, Sol. State Commun. 7, 415 (1969).

[9] R. Blinc, M. Luzar, M. Vilfan, and M. Burgar, J. Chem. Phys. 63, 3445 (1975).
[10] J. Freed, J. Chem. Phys. 66, 4183 (1977).

[11] H. Pfeifer, Ann. Phys. 7, 1 (1961).

[12] S. Zumer and M. Vilfan, Phys. Rev. A 17, 424 (1978).

[13] M. Vilfan and S. Žumer, Phys. Rev. A 21, 672 (1980).

[14] D. Woessner, J. Chem. Phys. 37, 647 (1962).

[15] F. Winter and R. Kimmich, Mol. Phys. 45, 33 (1982).

[16] R. Kimmich, F. Winter, W. Nusser, and K. Spohn, J. Magn. Reson. 68, 263 (1986).

[17] B. Cabane and W. Clark, Phys. Rev. Lett. 25, 91 (1970).

[18] W. Krigbaum, Y. Chatani, and P. Barber, Acta Cryst. B 26, 97 (1970).

[19] J. Seliger, R. Osredkar, M. Mali, and R. Blinc, J. Chem. Phys. 65, 2887 (1976).

[20] R. Lotfullin and G. Semin, Adv. Nuclear Quadrupole Resonance 2, 1 (1975).

[21] R. Lotfullin and G. Semin, J. Strukt. Khim. 9, 813 (1968). 